\title{
Opioid and benzodiazepine dispensing and co-dispensing patterns among commercially insured pregnant women in the United States, 2007-2015
}

Danya M. Qato ${ }^{1,2^{*}}$ (D) and Aakash Bipin Gandhi ${ }^{1}$

\begin{abstract}
Background: Little is known about benzodiazepine and opioid-benzodiazepine co-dispensing patterns among pregnant women. Understanding these patterns is necessary to mitigate high-risk medication use during pregnancy. Our objective in this analysis was to evaluate opioid and benzodiazepine dispensing and co-dispensing patterns among commercially insured pregnant women in the United States.

Methods: This retrospective study used a 10\% random sample of commercially insured enrollees from the IQVIA ${ }^{\mathrm{TM}}$ Adjudicated Health Plan Claims Data from 2007 to 2015. The study included women (12-55 years of age) with completed pregnancies who had continuous medical and prescription drug coverage from 3 months prior to the date of conception through 3 months post-delivery. We estimated the prevalence of opioid and benzodiazepine dispensing and co-dispensing before, during, and after pregnancy, and evaluated trends in dispensing patterns across the study period (2007-2015) using Cochrane-Armitage tests. Chi-square tests were used to examine differences in demographic and clinical characteristics by dispensing and co-dispensing patterns. Among women that received an opioid or benzodiazepine during pregnancy, logistic regression models were used to quantify the association between sample characteristics and dispensing patterns (co-dispensing vs single dispensing).

Results: Of 168,025 pregnant women that met our inclusion criteria, 10.1\% received at least one opioid and 2.0\% received at least one benzodiazepine during pregnancy, while $0.5 \%$ were co-dispensed these drugs. During the study period (2007 vs 2015), prevalence of opioid dispensing during pregnancy decreased from 11.2 to $8.6 \%$ $(p<0.01)$; while benzodiazepine dispensing increased from 1.3 to $2.9 \%(p<0.01)$, and the prevalence of codispensing, while low and stable, increased slightly from 0.39 to $0.44 \%(p<0.01)$. Older age, a higher comorbidity burden, pain diagnosis, anxiety diagnosis, and alcohol, tobacco, and drug use disorders, were all associated with an increased odds of co-dispensing during pregnancy.

(Continued on next page)
\end{abstract}

\footnotetext{
* Correspondence: dqato@rx.umaryland.edu

${ }^{1}$ Department of Pharmaceutical Health Services Research, University of

Maryland School of Pharmacy, Baltimore, MD, USA

${ }^{2}$ Department of Epidemiology and Public Health, University of Maryland

School of Medicine, Baltimore, MD, USA
}

(c) The Author(s). 2021 Open Access This article is licensed under a Creative Commons Attribution 4.0 International License, which permits use, sharing, adaptation, distribution and reproduction in any medium or format, as long as you give appropriate credit to the original author(s) and the source, provide a link to the Creative Commons licence, and indicate if changes were made. The images or other third party material in this article are included in the article's Creative Commons licence, unless indicated otherwise in a credit line to the material. If material is not included in the article's Creative Commons licence and your intended use is not permitted by statutory regulation or exceeds the permitted use, you will need to obtain permission directly from the copyright holder. To view a copy of this licence, visit http://creativecommons.org/licenses/by/4.0/. The Creative Commons Public Domain Dedication waiver (http://creativecommons.org/publicdomain/zero/1.0/) applies to the data made available in this article, unless otherwise stated in a credit line to the data. 
(Continued from previous page)

Conclusions: This study provides evidence that while opioid dispensing during pregnancy has decreased in the past decade, benzodiazepine dispensing has increased. The prevalence of opioid-benzodiazepine co-dispensing was rare and remained fairly stable during our study period. Those co-dispensed both drugs had a higher prevalence of adverse birth outcomes. Further research to establish the potentially causal relationship between opioid and benzodiazepine co-dispensing and adverse birth outcomes should be undertaken.

Keywords: Pregnant women, Commercially insured, Opioid, Benzodiazepine, Dispensing, Trend analysis

\section{Background}

The use and co-use of opioids and benzodiazepines may pose significant health risks to pregnant women. Opioid use during pregnancy is associated with obstetric complications like spontaneous abortions and preeclampsia, as well as adverse birth outcomes such as neural tube defects, gastroschisis, and neonatal abstinence syndrome (NAS) [1-3]. While benzodiazepines may have health benefits that outweigh their risks [4], evidence on the safety of benzodiazepine use during pregnancy is mixed [5-9]. Some studies report that benzodiazepine use during pregnancy is associated with preterm birth, low birth weight, as well as congenital malformations [5-7], while other studies have found no impact of in-utero exposure to benzodiazepines on birthweight or neurodevelopment $[8,9]$. The co-use of opioids and benzodiazepines in pregnancy is associated with an increased risk of maternal overdose and mortality, increased risk and severity of NAS, and longer newborn hospital stays [10-13].

The prevalence of opioid dispensing during pregnancy in the United States (U.S.) is well understood [14, 15]. Most recently, a study analyzing a nationally representative sample of commercially insured women reported a decreasing trend in opioid dispensing during pregnancy (14.9\% in 2005 to $12.9 \%$ in 2011). However, the overall prevalence of dispensing was observed to be high at an average of $14.4 \%$ across the study period [14].

In contrast to opioids, the epidemiology of benzodiazepine dispensing during pregnancy has been less explored. The National Birth Defects Prevention Study, conducted across 10 U.S. states between 1997 and 2011, found that the prevalence of self-reported benzodiazepine use during pregnancy was rare (0.8\%) [7]. Another study, in a commercially insured population, reported the prevalence of anxiolytic (benzodiazepine or Z-drug) dispensing during pregnancy to be $3.9 \%$ between 2006 and 2011 [16]. These prior studies have limited generalizability because of the clinically unique and/or geographically distinct study samples used, or are estimates of overall anxiolytic prescribing and not specifically of benzodiazepines $[7,16]$.

There is also limited literature on the co-dispensing of opioids and benzodiazepines during pregnancy. Investigating such dispensing patterns is especially important given that in 2016, the U.S. Food and Drug
Administration (FDA) announced labelling changes, cautioning against the dangers of using opioids and benzodiazepines concomitantly [17]. Additionally, despite the risk for fatal overdose, recent evidence in the general population has found that over one-third of benzodiazepine prescriptions involved an overlapping opioid prescription [18].

In light of the research gaps and potential risks associated with use and co-use during pregnancy, we sought to quantify the prevalence and temporal trends of opioid and benzodiazepine dispensing and co-dispensing before, during, and after pregnancy, and by birth outcomes. Further, we examined differences in demographic and clinical characteristics of women in our sample by mutually exclusive opioid and benzodiazepine dispensing patterns.

\section{Methods \\ Data source}

In this retrospective study, we utilized a $10 \%$ random sample of primarily commercially insured (97\%) enrollees in the IQVIA $^{\mathrm{Tm}}$ Adjudicated Health Plan Claims Data. While we only had access to $10 \%$ of the full data available through IQVIA, the sample was randomly generated by the vendor. Thus, to the extent possible, we believe the prevalence estimates are representative of the entire sample. IQVIA ${ }^{\mathrm{Tm}}$ data is a longitudinal dataset and includes medical, pharmacy, and eligibility information from over 70 contributing health plans and self-insured employer groups throughout the U.S. for more than 140 million unique enrollees younger than 65 years of age.

\section{Study population}

We identified women (12-55 years of age at date of delivery) with a completed pregnancy between 2007 and 2015. We used a previous algorithm (using ICD-9-CM diagnostic and procedure codes) for the identification of completed pregnancies, their categorization by birth outcomes (full-term birth, preterm birth, postterm birth, stillbirth), and the assignment of gestational age estimates [19]. We excluded women with spontaneous abortions or terminated pregnancies. In order to analyze drug dispensing patterns, beneficiaries were required to have continuous medical and prescription drug coverage 
from 3 months prior to the date of conception through 3 months post-delivery, allowing for a one-month gap in coverage. Only the first eligible delivery episode for each pregnant woman was considered for analysis.

\section{Definition of dispensing periods}

We examined opioid and benzodiazepine dispensing and co-dispensing in each of the following periods: preconception (90-day period prior to the estimated date of conception); trimester 1 (estimated conception date through day 90 of pregnancy); trimester 2 (following 90 days); trimester 3 (beginning 181 days after estimated date of conception to date of delivery); and post-delivery (90-day period after the date of delivery).

\section{Definition of primary exposures}

Our principal exposures of interest were opioid and benzodiazepine dispensing and co-dispensing. We only included opioids and benzodiazepines in the Centers for Disease Control and Prevention's (CDC) guidance for analyzing opioid prescription data [20]. We excluded buprenorphine-naloxone combination products from the analysis. We used pharmacy claims to identify opioid and benzodiazepine dispensing dates within each time period of interest. The co-dispensing of opioids and benzodiazepines was defined based on prior literature as having at least a 1 day overlap in days' supply of both drugs during any of the designated time periods [21].

\section{Demographic and clinical characteristics}

The following demographic and clinical characteristics were determined on the date of delivery: age, region of residence, cesarean delivery. The following clinical characteristics were determined, using ICD-9-CM codes, between the start of the pre-conception period through the date of delivery: back pain, abdominal pain, arthritis pain, headache, cancer, obesity, anxiety, alcohol use disorder, tobacco use disorder, and drug use disorder. Finally, an obstetric comorbidity index, developed and validated previously, was used to determine the pre-pregnancy comorbidity burden over the same period [22].

\section{Statistical analysis}

Descriptive analyses using chi-square tests were used to estimate demographic and clinical characteristics for the total sample and by each birth outcome. We estimated the prevalence of prescription opioid and benzodiazepine dispensing and co-dispensing before, during, and after pregnancy, and evaluated temporal trends during the study period as defined by delivery year using Cochrane-Armitage tests for trends.

Differences in beneficiary characteristics were also examined across five mutually exclusive groups depending on dispensing patterns during pregnancy: co-dispensed opioid-benzodiazepine, dispensed opioid only, dispensed benzodiazepine only, no opioid or benzodiazepine dispensing, and opioid-benzodiazepine dispensing but not co-dispensed. The latter group was defined as individuals who were dispensed both benzodiazepines and opioids at some point during pregnancy, but not concomitantly. Among women that received an opioid or benzodiazepine during pregnancy, a multivariable logistic regression was used to quantify the association between the sample characteristics and dispensing patterns. In this logistic regression analysis we compared those with co-dispensing vs single dispensing (opioid only, benzodiazepine only, opioid and benzodiazepine but not co-dispensed). The sample characteristics included in the model were age at delivery, region of residence, delivery year, comorbidity index score, obesity, cancer, any pain diagnosis (back pain, abdominal pain, arthritis pain, or headache), anxiety diagnosis, and alcohol, tobacco, or drug disorder diagnosis. All analyses were conducted using SAS version 9.4 and statistical significance was set at $\alpha=0.05$. The study received Institutional Review Board approval by the University of Maryland, Baltimore.

\section{Results}

We identified 168,025 completed pregnancies over a nine-year period that met our eligibility criteria. Table 1 summarizes the demographic and clinical characteristics of the total sample and by individual birth outcomes. Birth outcomes in our sample were distributed as follows: full-term birth (79.4\%), postterm birth (12.4\%), preterm birth (7.2\%), and stillbirth (1\%). Relative to those with a full-term birth, women who experienced a preterm birth or a stillbirth were older at delivery, had a higher comorbidity burden, and displayed a higher prevalence of alcohol, tobacco, and drug use disorders.

Figure 1 displays prevalence estimates of opioid and benzodiazepine dispensing and co-dispensing during pregnancy, in the total sample and by each birth outcome. Overall, $10.1 \%$ of the total sample was dispensed at least one opioid during pregnancy. Prevalence of opioid dispensing varied significantly $(p<0.01)$ by birth outcome: stillbirth (14.3\%), preterm birth (13.5\%), fullterm birth (10.2\%), and postterm birth (7.3\%). Two percent of the total sample was dispensed at least one benzodiazepine during pregnancy. The prevalence of benzodiazepine dispensing varied significantly $(p<0.01)$ by birth outcome: stillbirth (4.8\%), preterm birth (3.5\%), full-term birth (1.9\%), and postterm birth (1.4\%). Finally, $0.5 \%$ of the total sample was co-dispensed opioids and benzodiazepines during pregnancy. Prevalence of codispensing also varied significantly $(p<0.01)$ by birth 
Table 1 Demographic and clinical characteristics of commercially insured pregnant women (12-55 years of age) in the United States by birth outcome, 2007-2015

\begin{tabular}{|c|c|c|c|c|c|c|c|c|c|c|c|}
\hline & \multicolumn{2}{|c|}{$\begin{array}{l}\text { Total population } \\
N=168,025 \\
(100 \%)\end{array}$} & \multicolumn{2}{|c|}{$\begin{array}{l}\text { Full-term birth } \\
N=133,459 \\
(79.4 \%)\end{array}$} & \multicolumn{2}{|c|}{$\begin{array}{l}\text { Preterm birth } \\
N=12,166 \\
(7.2 \%)\end{array}$} & \multicolumn{2}{|c|}{$\begin{array}{l}\text { Postterm birth } \\
N=20,802 \\
(12.4 \%)\end{array}$} & \multicolumn{2}{|c|}{$\begin{array}{l}\text { Stillbirth } \\
N=1598 \\
(1.0 \%)\end{array}$} & \multirow[t]{2}{*}{$P$-value } \\
\hline & $\mathrm{N}$ & Col\% & $\mathbf{N}$ & Col\% & $\mathbf{N}$ & Col\% & $\mathbf{N}$ & Col\% & $\mathbf{N}$ & Col\% & \\
\hline Age category, years & & & & & & & & & & & $<0.01$ \\
\hline $12-17$ & 1920 & 1.1 & 1452 & 1.1 & 165 & 1.4 & 280 & 1.3 & 23 & 1.4 & \\
\hline $18-25$ & 31,591 & 18.8 & 24,731 & 18.5 & 2276 & 18.7 & 4287 & 20.6 & 297 & 18.6 & \\
\hline $26-34$ & 97,905 & 58.3 & 77,734 & 58.3 & 6794 & 55.8 & 12,536 & 60.3 & 841 & 52.6 & \\
\hline$\geq 35$ & 36,609 & 21.8 & 29,542 & 22.1 & 2931 & 24.1 & 3699 & 17.8 & 437 & 27.4 & \\
\hline Region & & & & & & & & & & & $<0.01$ \\
\hline East & 35,220 & 21.0 & 27,042 & 20.2 & 2460 & 20.2 & 5404 & 25.9 & 314 & 19.6 & \\
\hline Midwest & 52,909 & 31.5 & 41,321 & 31.0 & 3584 & 29.5 & 7527 & 36.2 & 477 & 29.9 & \\
\hline South & 54,863 & 32.6 & 45,333 & 34.0 & 4293 & 35.3 & 4635 & 22.3 & 602 & 37.7 & \\
\hline West & 25,033 & 14.9 & 19,763 & 14.8 & 1829 & 15.0 & 3236 & 15.6 & 205 & 12.8 & \\
\hline Comorbidity score & & & & & & & & & & & $<0.01$ \\
\hline 0 & 79,460 & 47.3 & 61,669 & 46.2 & 4078 & 33.5 & 13,080 & 62.9 & 633 & 39.6 & \\
\hline 1 & 39,959 & 23.8 & 33,299 & 25.0 & 2075 & 17.1 & 4298 & 20.7 & 287 & 18.0 & \\
\hline$\geq 2$ & 48,606 & 28.9 & 38,491 & 28.8 & 6013 & 49.4 & 3424 & 16.4 & 678 & 42.4 & \\
\hline Cesarean delivery & & & & & & & & & & & $<0.01$ \\
\hline Yes & 40,749 & 24.3 & 33,412 & 25.0 & 8644 & 71.1 & 3615 & 17.4 & 200 & 12.5 & \\
\hline No & 127,276 & 75.7 & 100,047 & 75.0 & 3522 & 28.9 & 17,187 & 82.6 & 1398 & 87.5 & \\
\hline Obesity & & & & & & & & & & & $<0.01$ \\
\hline Yes & 12,708 & 7.6 & 9987 & 7.5 & 1147 & 9.4 & 1418 & 6.8 & 156 & 9.8 & \\
\hline No & 155,317 & 92.4 & 123,472 & 92.5 & 11,019 & 90.6 & 19,384 & 93.2 & 1442 & 90.2 & \\
\hline Cancer & & & & & & & & & & & 0.49 \\
\hline Yes & 1270 & 0.8 & 1000 & 0.8 & 106 & 0.9 & 152 & 0.7 & 12 & 0.7 & \\
\hline No & 166,755 & 99.2 & 132,459 & 99.2 & 12,060 & 99.1 & 20,650 & 99.3 & 1586 & 99.3 & \\
\hline Back pain & & & & & & & & & & & $<0.01$ \\
\hline Yes & 30,441 & 18.1 & 24,121 & 18.1 & 2296 & 18.9 & 3785 & 18.2 & 239 & 15.0 & \\
\hline No & 137,584 & 81.9 & 109,338 & 81.9 & 9870 & 81.1 & 17,017 & 81.8 & 1359 & 85.0 & \\
\hline Abdominal pain & & & & & & & & & & & $<0.01$ \\
\hline Yes & 37,766 & 22.5 & 29,851 & 22.4 & 3491 & 28.7 & 4045 & 19.4 & 379 & 23.7 & \\
\hline No & 130,259 & 77.5 & 103,608 & 77.6 & 8675 & 71.3 & 16,757 & 80.6 & 1219 & 76.3 & \\
\hline Arthritis pain & & & & & & & & & & & $<0.01$ \\
\hline Yes & 35,090 & 20.9 & 27,645 & 20.7 & 2653 & 21.8 & 4478 & 21.5 & 314 & 19.7 & \\
\hline No & 132,935 & 79.1 & 105,814 & 79.3 & 9513 & 78.2 & 16,324 & 78.5 & 1284 & 80.3 & \\
\hline Headache & & & & & & & & & & & $<0.01$ \\
\hline Yes & 6564 & 3.9 & 5171 & 3.9 & 575 & 4.7 & 748 & 3.6 & 70 & 4.4 & \\
\hline No & 161,461 & 96.1 & 128,288 & 96.1 & 11,591 & 95.3 & 20,054 & 96.4 & 1528 & 95.6 & \\
\hline Anxiety disorders & & & & & & & & & & & $<0.01$ \\
\hline Yes & 10,059 & 6.0 & 7961 & 6.0 & 863 & 7.1 & 1121 & 5.4 & 114 & 7.1 & \\
\hline No & 157,966 & 94.0 & 125,498 & 94.0 & 11,303 & 92.9 & 19,681 & 94.6 & 1484 & 92.9 & \\
\hline Alcohol use disorder & & & & & & & & & & & 0.01 \\
\hline Yes & 295 & 0.2 & 214 & 0.2 & 29 & 0.2 & 46 & 0.2 & 6 & 0.4 & \\
\hline No & 167,730 & 99.8 & 133,245 & 99.8 & 12,137 & 99.8 & 20,756 & 99.8 & 1592 & 99.6 & \\
\hline
\end{tabular}


Table 1 Demographic and clinical characteristics of commercially insured pregnant women (12-55 years of age) in the United States by birth outcome, 2007-2015 (Continued)

\begin{tabular}{|c|c|c|c|c|c|c|c|c|c|c|c|}
\hline & \multicolumn{2}{|c|}{$\begin{array}{l}\text { Total population } \\
N=168,025 \\
(100 \%)\end{array}$} & \multicolumn{2}{|c|}{$\begin{array}{l}\text { Full-term birth } \\
N=133,459 \\
(79.4 \%)\end{array}$} & \multicolumn{2}{|c|}{$\begin{array}{l}\text { Preterm birth } \\
N=12,166 \\
(7.2 \%)\end{array}$} & \multicolumn{2}{|c|}{$\begin{array}{l}\text { Postterm birth } \\
N=20,802 \\
(12.4 \%)\end{array}$} & \multicolumn{2}{|c|}{$\begin{array}{l}\text { Stillbirth } \\
N=1598 \\
(1.0 \%)\end{array}$} & \multirow[t]{2}{*}{$P$-value } \\
\hline & $\mathbf{N}$ & Col\% & $\mathrm{N}$ & Col\% & $\mathrm{N}$ & Col\% & $\mathrm{N}$ & Col\% & $\mathbf{N}$ & Col\% & \\
\hline Tobacco use disorder & & & & & & & & & & & $<0.01$ \\
\hline Yes & 7614 & 4.5 & 6047 & 4.5 & 720 & 5.9 & 769 & 3.7 & 78 & 4.9 & \\
\hline No & 160,411 & 95.5 & 127,412 & 95.5 & 11,446 & 94.1 & 20,033 & 96.3 & 1520 & 95.1 & \\
\hline Drug use disorder & & & & & & & & & & & 0.02 \\
\hline Yes & 342 & 0.2 & 249 & 0.2 & 30 & 0.3 & 58 & 0.3 & 5 & 0.3 & \\
\hline No & 167,683 & 99.8 & 133,210 & 99.8 & 12,136 & 99.7 & 20,744 & 99.7 & 1593 & 99.7 & \\
\hline
\end{tabular}

outcome: stillbirth $(1.2 \%)$, preterm birth $(1.1 \%)$, full-term birth $(0.5 \%)$, and postterm birth $(0.3 \%)$.

Figure 2 reflects temporal trends in opioid and benzodiazepine dispensing as well as co-dispensing during pregnancy across each year of the study for the total sample. While there was a statistically significant decrease in the prevalence of opioid dispensing during pregnancy between 2007 and 2015 (11.2 to 8.6\%, $p<0.01$ ), benzodiazepine dispensing increased over the same period $(1.3$ to $2.9 \%, p<0.01)$. In addition, there was a statistically significant increase in opioidbenzodiazepine co-dispensing during pregnancy over the same period $(0.39 \%$ vs $0.44 \%, p=0.01)$. Additional file 1 reflects the statistically significant increasing trend in the prevalence of any pain diagnosis (back pain, abdominal pain, arthritis pain or headache), anxiety diagnosis, and cesarean delivery across the study period in the total sample. Additional file 2 and Additional file 3 present temporal trends for the five most commonly dispensed opioids and benzodiazepines, respectively, during pregnancy across the study period. Additional file 4 and Additional file 5 describe the prevalence of the five most commonly dispensed opioids and benzodiazepines, respectively, before, during, and after pregnancy.

Figures 3a-c display temporal trends in opioid and benzodiazepine dispensing and co-dispensing by delivery year and pregnancy period for the total sample. A significantly decreasing trend (2007 vs 2015) in opioid dispensing during the 1 st trimester $(4.6 \%$ vs $3.4 \%$, $p<0.01)$, 2nd trimester ( $4.3 \%$ vs $3 \%, p<0.01)$, and 3rd trimester $(4.8 \%$ vs $3.7 \%, p<0.01)$ was observed across the study period. Conversely, prevalence of opioid dispensing during the post-delivery period increased over the same period ( $45.0 \%$ vs $48.3 \%, p<0.01$ ) (Fig. 3a).

The prevalence of benzodiazepine dispensing increased (2007 vs 2015$)$ in the pre-conception $(2.3 \%$ vs $3.8 \%$, $p<0.01)$, 1 st trimester (1.1\% vs $2.1 \%, p<0.01$ ), and 2 nd trimester $(0.3 \%$ vs $0.5 \%, p<0.01)$ periods. Conversely, dispensing in the post-delivery period decreased over a similar time period $(2.4 \%$ vs $2.1 \%, p=0.02)$ (Fig. $3 \mathrm{~b})$. The

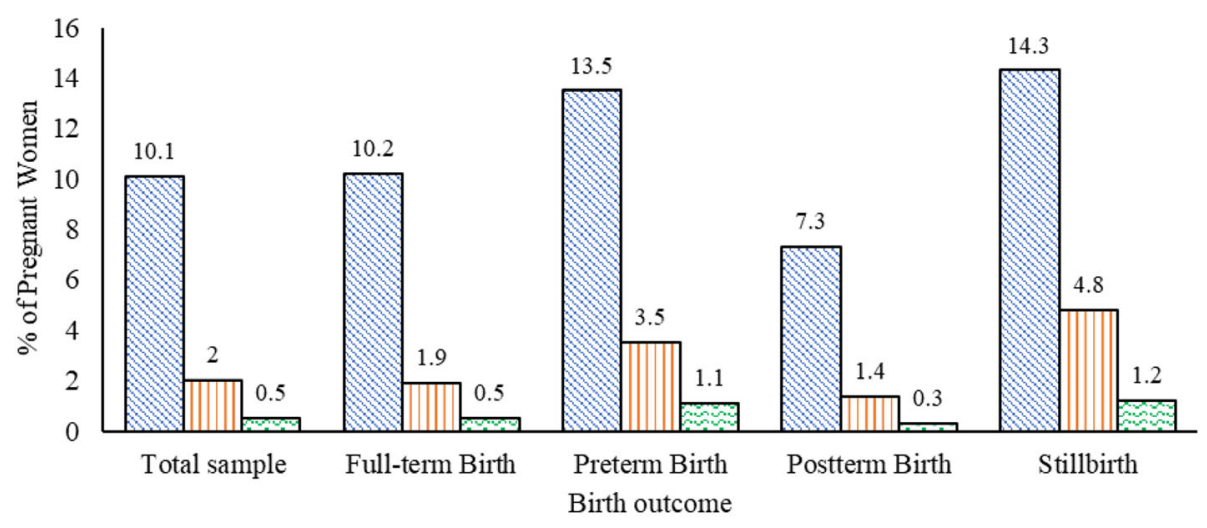

口Opioid dispensing $\square$ Benzodiazepine dispensing $\square$ Opioid-benzodiazepine co-dispensing

Fig. 1 Opioid and benzodiazepine dispensing and co-dispensing during pregnancy by birth outcome among commercially insured women in the United States, 2007-2015.

Statistically significant difference $(p<0.01)$ for opioid dispensing, benzodiazepine dispensing, and opioid-benzodiazepine co-dispensing by birth outcome 


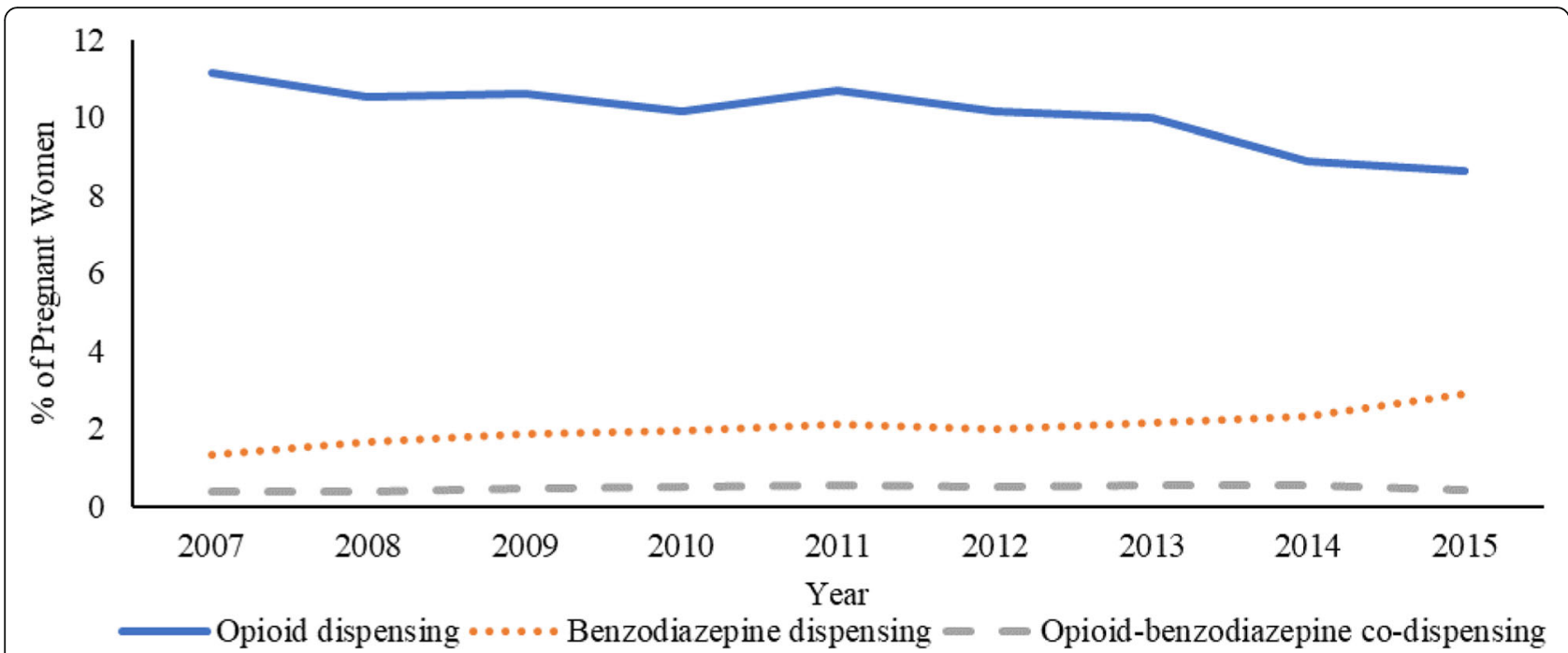

Fig. 2 Temporal trends in opioid and benzodiazepine dispensing and co-dispensing during pregnancy among commercially insured women in the United States, 2007-2015.

Statistically significant test of linear trend for opioid dispensing $(p<0.01)$, benzodiazepine dispensing $(p<0.01)$, and opioid-benzodiazepine co-dispensing ( $p=0.01)$

prevalence of opioid-benzodiazepine co-dispensing increased (2007 vs 2015) in the pre-conception $(0.5 \%$ vs $0.8 \%, p=0.01)$ and 1 st trimester $(0.3 \%$ vs $0.4 \%, p=0.01)$ periods (Fig. 3c). Additional file 6 describes trends (2007 vs 2015) in opioid dispensing and opioid-benzodiazepine co-dispensing patterns in the post-delivery period by mode of delivery (cesarean vs vaginal). Table 2 describes demographic and clinical characteristics of the sample by mutually exclusive opioid and benzodiazepine dispensing and co-dispensing patterns. Relative to those dispensed an opioid only, women co-dispensed opioids and benzodiazepines during pregnancy were older at birth, primarily resided in the south region of the U.S., had a higher comorbidity burden and displayed a higher prevalence of alcohol, tobacco, or drug use disorders.

Figure 4 presents the adjusted odds ratios (AOR) and $95 \%$ confidence intervals (CI) from the logistic regression model predicting opioid-benzodiazepine codispensing vs single dispensing. Determinants of codispensing during pregnancy include: older age, specifically women aged $\geq 35$ years compared to those $18-25$ years [AOR $(95 \% \mathrm{CI})$ : 2.57 (1.97, 3.34)]; residence in the south region of the U.S. compared to midwest region [AOR $1.65(1.36,1.99)]$; a higher comorbidity index score ( $\geq 2$ vs 0 ) [AOR 1.33 (1.10, 1.61)]; having a pain diagnosis [AOR 1.56 (1.32, 1.86)]; having an anxiety diagnosis [AOR 4.06 (3.49, 4.73)]; and having an alcohol, tobacco, or drug use disorder [AOR $1.93(1.60,2.30)]$.

\section{Discussion}

Over our 9-year study period, 1 in 10 and 1 in 50 pregnant women were dispensed an opioid and benzodiazepine, respectively, during pregnancy. We

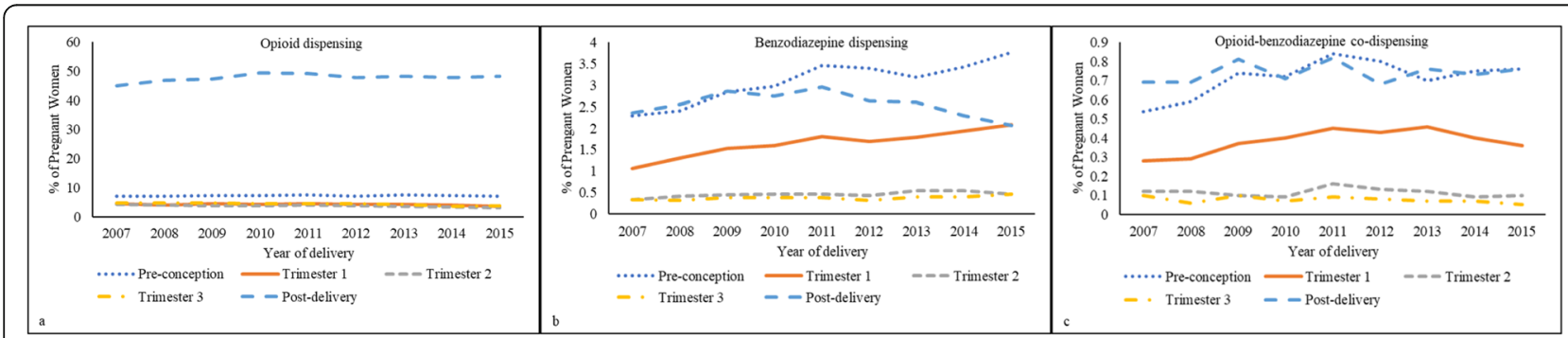

Fig. 3 Temporal trends in opioid and benzodiazepine dispensing and co-dispensing by pregnancy time periods among commercially insured women in the United States, 2007-2015.

Statistically significant test of linear trend for opioid dispensing during all time periods $(p<0.01)$; benzodiazepine dispensing during preconception $(p<0.01)$, 1st trimester $(p<0.01)$, 2nd trimester $(p<0.01)$, and post-delivery $(p<0.01)$ periods; and opioid-benzodiazepine co-dispensing during pre-conception $(p=0.01)$ and 1 st trimester $(p=0.01)$ periods 
Table 2 Demographic, clinical, and birth outcome characteristics of commercially insured pregnant women in the United States (12-55 years of age) by mutually exclusive opioid and benzodiazepine dispensing categories, 2007-2015

\begin{tabular}{|c|c|c|c|c|c|c|c|c|c|c|c|}
\hline & \multicolumn{2}{|c|}{$\begin{array}{l}\text { Co-dispensed } \\
\text { opioid- } \\
\text { benzodiazepine } \\
N=808 \\
(0.5 \%)\end{array}$} & \multicolumn{2}{|c|}{$\begin{array}{l}\text { Dispensed, not } \\
\text { co-dispensed, opi- } \\
\text { oid- } \\
\text { benzodiazepine } \\
N=476 \\
(0.3 \%)\end{array}$} & \multicolumn{2}{|c|}{$\begin{array}{l}\text { Dispensed } \\
\text { opioid only } \\
N=15,699 \\
(9.3 \%)\end{array}$} & \multicolumn{2}{|c|}{$\begin{array}{l}\text { Dispensed } \\
\text { benzodiazepine } \\
\text { only } \\
N=2035 \\
(1.2 \%)\end{array}$} & \multicolumn{2}{|c|}{$\begin{array}{l}\text { Not dispensed } \\
\text { opioid or } \\
\text { benzodiazepine } \\
N=149,007 \\
(88.7 \%)\end{array}$} & \multirow[t]{2}{*}{$P$-value } \\
\hline & $\mathrm{N}$ & $\%$ & $\mathrm{~N}$ & $\%$ & $\mathrm{~N}$ & $\%$ & $\mathrm{~N}$ & $\%$ & $\mathrm{~N}$ & $\%$ & \\
\hline Age category, years & & & & & & & & & & & $<0.01$ \\
\hline $12-25$ & 98 & 12.1 & 68 & 14.3 & 3723 & 23.7 & 251 & 12.3 & 29,371 & 19.7 & \\
\hline $26-34$ & 459 & 56.8 & 275 & 57.8 & 8805 & 56.1 & 1071 & 52.6 & 87,295 & 58.6 & \\
\hline$\geq 35$ & 251 & 31.1 & 133 & 27.9 & 3171 & 20.2 & 713 & 35.1 & 32,341 & 21.7 & \\
\hline Region & & & & & & & & & & & $<0.01$ \\
\hline East & 153 & 18.9 & 82 & 17.2 & 2322 & 14.8 & 480 & 23.6 & 32,183 & 21.6 & \\
\hline Midwest & 176 & 21.8 & 134 & 28.2 & 4799 & 30.6 & 589 & 28.9 & 47,211 & 31.7 & \\
\hline South & 349 & 43.2 & 215 & 45.2 & 6111 & 38.9 & 771 & 37.9 & 47,417 & 31.8 & \\
\hline West & 130 & 16.1 & 45 & 9.4 & 2467 & 15.7 & 195 & 9.6 & 22,196 & 14.9 & \\
\hline Comorbidity index & & & & & & & & & & & $<0.01$ \\
\hline 0 & 236 & 29.2 & 142 & 29.8 & 6438 & 41.0 & 673 & 33.0 & 71,971 & 48.3 & \\
\hline 1 & 197 & 24.4 & 111 & 23.3 & 3888 & 24.8 & 500 & 24.6 & 35,263 & 23.7 & \\
\hline$\geq 2$ & 375 & 46.4 & 223 & 46.9 & 5373 & 34.2 & 862 & 42.4 & 41,773 & 28.0 & \\
\hline Cesarean delivery & & & & & & & & & & & $<0.01$ \\
\hline Yes & 296 & 36.6 & 164 & 34.5 & 4484 & 28.6 & 638 & 31.4 & 35,167 & 23.6 & \\
\hline No & 512 & 63.4 & 312 & 65.5 & 11,215 & 71.4 & 1397 & 68.6 & 113,840 & 76.4 & \\
\hline Birth type & & & & & & & & & & & $<0.01$ \\
\hline Full-term birth & 601 & 74.4 & 366 & 76.9 & 12,625 & 80.4 & 1561 & 76.7 & 118,306 & 79.4 & \\
\hline Preterm birth & 132 & 16.3 & 56 & 11.8 & 1450 & 9.2 & 232 & 11.4 & 10,296 & 6.9 & \\
\hline Post term birth & 56 & 6.9 & 41 & 8.6 & 1427 & 9.1 & 197 & 9.7 & 19,081 & 12.8 & \\
\hline Stillbirth & 19 & 2.4 & 13 & 2.7 & 197 & 1.3 & 45 & 2.2 & 1324 & 0.9 & \\
\hline Obesity & & & & & & & & & & & $<0.01$ \\
\hline Yes & 100 & 12.4 & 67 & 14.1 & 1691 & 10.8 & 213 & 10.5 & 10,637 & 7.1 & \\
\hline No & 708 & 87.6 & 409 & 85.9 & 14,008 & 89.2 & 1822 & 89.5 & 138,370 & 92.9 & \\
\hline Cancer & & & & & & & & & & & $<0.01$ \\
\hline Yes & 13 & 1.6 & 9 & 1.9 & 172 & 1.1 & 30 & 1.5 & 1046 & 0.7 & \\
\hline No & 795 & 98.4 & 467 & 98.1 & 15,527 & 98.9 & 2005 & 98.5 & 147,961 & 99.3 & \\
\hline Back pain & & & & & & & & & & & $<0.01$ \\
\hline Yes & 384 & 47.5 & 197 & 41.4 & 5066 & 32.3 & 458 & 22.5 & 24,336 & 16.3 & \\
\hline No & 424 & 52.5 & 279 & 58.6 & 10,633 & 67.7 & 1577 & 77.5 & 124,671 & 83.7 & \\
\hline Abdominal pain & & & & & & & & & & & $<0.01$ \\
\hline Yes & 340 & 42.1 & 220 & 46.2 & 6790 & 43.3 & 590 & 29.0 & 29,826 & 20.0 & \\
\hline No & 468 & 57.9 & 256 & 53.8 & 8909 & 56.7 & 1445 & 71.0 & 119,181 & 80.0 & \\
\hline Arthritis pain & & & & & & & & & & & $<0.01$ \\
\hline Yes & 372 & 46.0 & 198 & 41.6 & 5102 & 32.5 & 604 & 29.7 & 28,814 & 19.3 & \\
\hline No & 436 & 54.0 & 278 & 58.4 & 10,597 & 67.5 & 1431 & 70.3 & 120,193 & 80.7 & \\
\hline Headache & & & & & & & & & & & $<0.01$ \\
\hline Yes & 149 & 18.4 & 70 & 14.7 & 1678 & 10.7 & 124 & 6.1 & 4543 & 3.1 & \\
\hline No & 659 & 81.6 & 406 & 85.3 & 14,021 & 89.3 & 1911 & 93.9 & 144,464 & 96.9 & \\
\hline
\end{tabular}


Table 2 Demographic, clinical, and birth outcome characteristics of commercially insured pregnant women in the United States (12-55 years of age) by mutually exclusive opioid and benzodiazepine dispensing categories, 2007-2015 (Continued)

\begin{tabular}{|c|c|c|c|c|c|c|c|c|c|c|c|}
\hline & \multicolumn{2}{|c|}{$\begin{array}{l}\text { Co-dispensed } \\
\text { opioid- } \\
\text { benzodiazepine } \\
N=808 \\
(0.5 \%)\end{array}$} & \multicolumn{2}{|c|}{$\begin{array}{l}\text { Dispensed, not } \\
\text { co-dispensed, opi- } \\
\text { oid- } \\
\text { benzodiazepine } \\
N=476 \\
(0.3 \%) \\
\end{array}$} & \multicolumn{2}{|c|}{$\begin{array}{l}\text { Dispensed } \\
\text { opioid only } \\
N=15,699 \\
(9.3 \%)\end{array}$} & \multicolumn{2}{|c|}{$\begin{array}{l}\text { Dispensed } \\
\text { benzodiazepine } \\
\text { only } \\
N=2035 \\
(1.2 \%)\end{array}$} & \multicolumn{2}{|c|}{$\begin{array}{l}\text { Not dispensed } \\
\text { opioid or } \\
\text { benzodiazepine } \\
N=149,007 \\
(88.7 \%)\end{array}$} & \multirow[t]{2}{*}{$P$-value } \\
\hline & $\mathrm{N}$ & $\%$ & $\mathbf{N}$ & $\%$ & $\mathrm{~N}$ & $\%$ & $\mathrm{~N}$ & $\%$ & $\mathrm{~N}$ & $\%$ & \\
\hline Anxiety disorders & & & & & & & & & & & $<0.01$ \\
\hline Yes & 325 & 40.2 & 198 & 58.4 & 1338 & 8.5 & 855 & 42.0 & 7343 & 4.9 & \\
\hline No & 483 & 59.8 & 278 & 41.6 & 14,361 & 91.5 & 1180 & 58.0 & 141,664 & 95.1 & \\
\hline Alcohol, tobacco, or drug use disorder & & & & & & & & & & & $<0.01$ \\
\hline Yes & 159 & 19.7 & 69 & 14.5 & 1659 & 10.6 & 202 & 9.9 & 6058 & 4.1 & \\
\hline No & 649 & 80.3 & 407 & 85.5 & 14,040 & 89.4 & 1833 & 90.1 & 142,949 & 95.9 & \\
\hline
\end{tabular}

observed a decreasing trend in opioid dispensing and an increasing trend in benzodiazepine dispensing during pregnancy. The prevalence of opioidbenzodiazepine co-dispensing during pregnancy was rare and remained stable over time. Sample characteristics such as older age, geographic residence, a higher comorbidity index score, presence of a pain diagnosis, presence of an anxiety diagnosis, and presence of alcohol, tobacco, and drug use disorders were significantly associated with opioid-benzodiazepine codispensing during pregnancy. Women who received both drugs had a higher prevalence of adverse birth outcomes compared to those dispensed opioids or benzodiazepines alone.

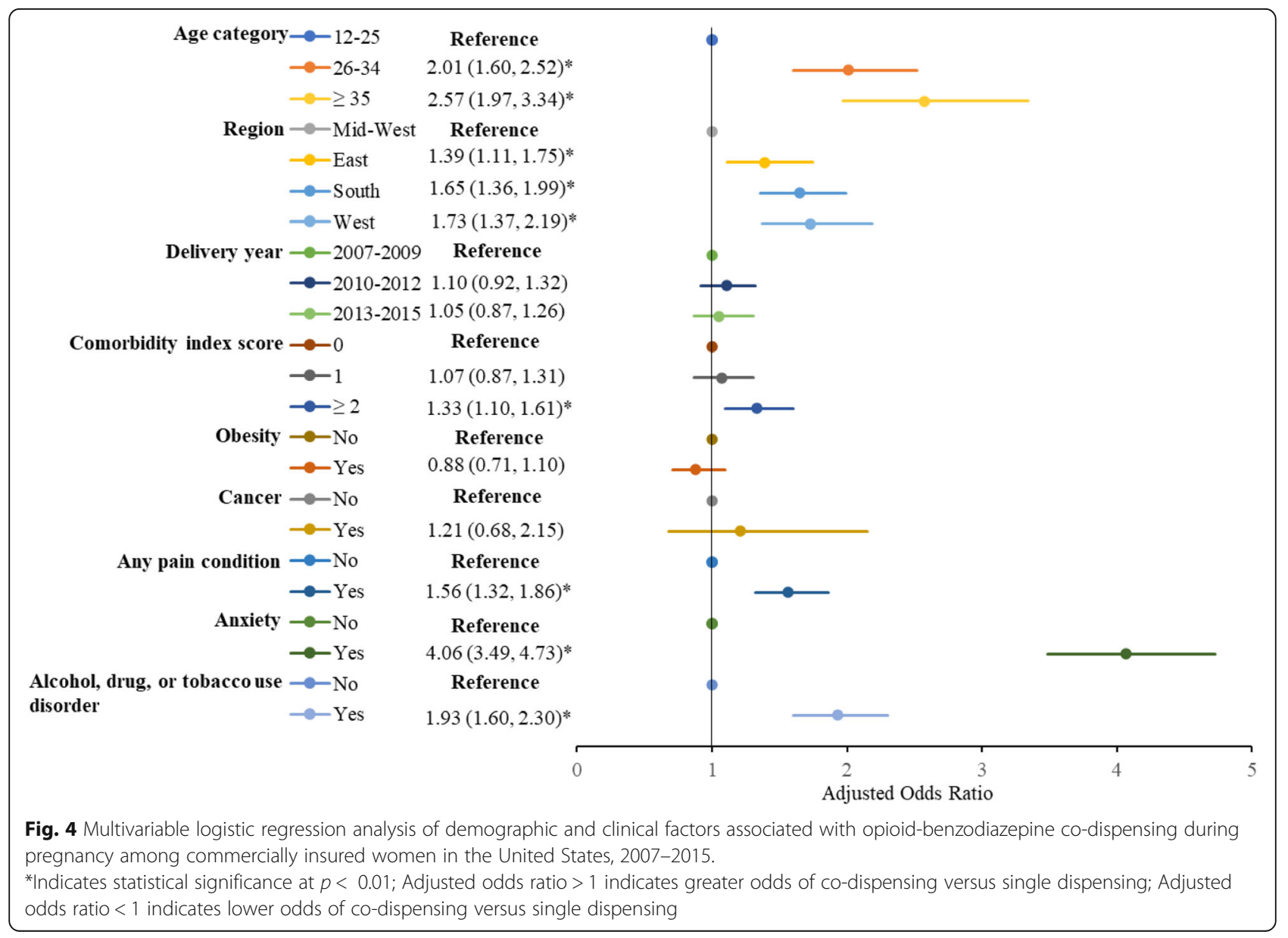


Our estimated prevalence of opioid dispensing during pregnancy of $10.1 \%$, is lower than that previously reported in a commercially insured population $(14.4 \%)$ in an earlier time period (2005-2011) [14]. Conversely, the prevalence of opioid dispensing in our study was higher than that observed in a European population in an earlier time period. A prior study using the linked Norwegian Prescription-Birth Registry Database reported the prevalence of opioid dispensing to be 3\% during pregnancy between 2004 and 2009 [23]. Our study findings thus reflect the decreasing trend in opioid use in the general U.S. population since 2012 [24]. Of note, despite the decrease in opioid dispensing, we found an increasing trend in pain diagnosis during the study period. The decreasing trend in opioid dispensing may thus be explained, in part, by the concerted federal, state, and local public health and managed care initiatives which have targeted the reduction of opioid prescribing rates in the general population in recent years [25-27]. Further, opioid dispensing during the post-delivery period rose significantly during our study period (2007-2015). This may be attributable to the overall increase (19.8 to $23.9 \%$ ) in cesarean deliveries in our sample as well as in the U.S during our study period [28].

Compared to our estimates, the National Birth Defects Prevention (NBDP) study reported a lower prevalence of self-reported benzodiazepine use during pregnancy (0.8\%) between 1997 and 2011 [7]. Notably, the NBDP study excluded states such as Alabama, Tennessee, and West Virginia, reported to have comparatively higher benzodiazepine prescribing rates than the general U.S. population [29]. Our estimate (2\%) for benzodiazepine dispensing during pregnancy was similar to that (1.9\%) reported in a prior meta-analysis which included 32 studies across 28 countries [30]. In sharp contrast to trends in opioid use, the prevalence of benzodiazepine dispensing during pregnancy more than doubled during our study period. Anxiety, the most frequent condition for which benzodiazepines are prescribed, is common and its prevalence is increasing among pregnant women [31]. Not surprisingly then, analogous to the trend in benzodiazepine dispensing, the prevalence of anxiety in our sample doubled (4.0 to $8.3 \%$ ) during the study period. Benzodiazepine dispensing during preconception and all three trimesters also rose significantly during our study period, again, likely reflective of the overall increase in prevalence of anxiety diagnoses.

We found that the prevalence of opioidbenzodiazepine co-dispensing during pregnancy was rare $(0.5 \%)$. One study, based on self-reported substance use among pregnant women admitted to opioid use disorder (OUD) treatment facilities, found that concomitant benzodiazepine use ranged from 3 to $13 \%$ depending on region of residence in the U.S. [32]. However, these individuals represent a subgroup with increased use patterns not representative of the general pregnant population. Another study estimated that the proportion of commercially insured individuals prescribed an opioid who were also prescribed a benzodiazepine in the general population (i.e. including men and non-pregnant women) was $17 \%$ in 2013 [21].

Not unexpectedly, while co-dispensing during pregnancy in our study was rare, variation was observed by birth outcomes. Pregnancies ending in preterm birth and stillbirth had 2- and 3-times higher prevalence of co-dispensing, respectively, compared to full-term birth. This finding should be considered with caution, as we did not study the causal association between codispensing and birth outcomes. Previous reports about the potential detrimental effects of these drugs on maternal and fetal health have been noted however [1-3, $5-7,9]$. Further, these findings are of concern given recent evidence that co-exposure to benzodiazepines and opioids increases the risk and severity of NAS $[10,11]$.

We found stable rates of co-dispensing during our study period. Though this cannot be attributed to the CDC guidelines and FDA drug labelling changes cautioning against co-use which were introduced later in 2016 [17, 33], it may be ascribed to similar guidelines developed earlier by the American Geriatrics Society and the American Society of Interventional Pain Physicians [34, 35]. While these guidelines are not specific to pregnant women, they helped create awareness of the risks of combined use, among both prescribers as well as patients.

In our sample and relative to those dispensed an opioid only, women co-dispensed opioids and benzodiazepines during pregnancy were older at birth, had a higher comorbidity burden, and displayed a higher prevalence of alcohol, tobacco, and drug use disorders. These findings are in line with a prior study analyzing a publicly insured U.S. sample of pregnant women co-prescribed opioids and psychotropics [10]. Similarly, our results from the logistic regression found that older age, geographic residence, a higher comorbidity index score, and presence of alcohol, tobacco, and drug use disorders were significantly associated with opioid-benzodiazepine co-dispensing (vs single dispensing of either product) during pregnancy. Pain and anxiety diagnosis were also found to be associated with co-dispensing during pregnancy.

Geographically, we found a higher prevalence of opioid and benzodiazepine dispensing and co-dispensing in the south compared to other U.S. regions. Prior studies have reported opioid prescribing rates are highest among commercially insured women of reproductive age residing in the south [36]. While similar estimates for benzodiazepine prescribing among pregnant women are not known, our results are consonant with prior findings of higher benzodiazepine prescribing rates in the south 
among commercially insured adults as compared to other U.S. regions [29]. Further, our findings reaffirm conclusions from a prior study which also found higher couse of opioids and benzodiazepines in the south among pregnant women entering OUD treatment facilities [32].

The increasing use of benzodiazepines is compelling to consider as a parallel problem to the opioid crisis [37]. Despite their associated risks, benzodiazepine use in vulnerable populations such as pregnant women has received little attention among clinicians, policymakers, and educators. Given the persistent co-use of opioids and benzodiazepines in the general population, as evidenced by recent research [18], our findings underscore the need to expand the focus of educational and intervention programs aimed at improving safe medication use among pregnant women beyond opioids. While opioids have become the focus of such programs, their aims should also extend to promoting prudent use of benzodiazepines and mitigating co-use of benzodiazepines and opioids.

Given the potential harmful effects of benzodiazepines on maternal and fetal health, our findings also highlight the need for non-pharmacological interventions to help relieve anxiety during pregnancy [38]. Removing barriers to access and improving insurance reimbursement for these interventions may help mitigate potentially inappropriate benzodiazepine prescribing in this population.

Given the lack of research on the safety of benzodiazepine use during pregnancy, our findings indicate several directions for future research. First, there is a need for additional studies to confirm the generalizability of our results in publicly insured and uninsured pregnant women. Second, future research should extend beyond dispensing patterns, to understand specific benzodiazepine drug doses or combinations that best balance the benefits of managing anxiety with the potential risk for adverse maternal and fetal outcomes. Third, in addition to prescribing guidelines and education, there is a need to evaluate additional strategies to manage or reduce the dispensing and co-dispensing of these drugs. Such strategies may include addressing benzodiazepine tapering or dose modification during pregnancy $[39,40]$, improving treatment of comorbid behavioral and mental health issues, and testing the comparative effectiveness of non-pharmacologic interventions. A comprehensive analysis of additional strategies will better inform policy and program development aimed at improving safe medication use during pregnancy.

Our study had several strengths. Our national sample allowed us to investigate opioid and benzodiazepine dispensing patterns in a cohort of 168,025 completed pregnancies sampled from over 70 commercial health plans across the U.S., with fully adjudicated medical and pharmacy claims. Whereas prior studies were mainly limited to live births, we included all completed pregnancy birth outcomes. Our study period was expansive and spanned 9 years, allowing for the examination of time trends in patterns before, during, and after pregnancy. Our focus on a commercially insured population allowed for a comprehensive analysis of prescribing practices in this population.

The present study had certain limitations. Importantly, our findings for the descriptive and trend analysis should be considered in light of the large sample size, which may yield statistically significant $p$-values for results that may not be of clinical significance. Further, our findings may not be generalizable to a large proportion of pregnant women who are uninsured or publicly insured. In addition, our findings are based on pharmacy dispensing claims that may not necessarily reflect use or prescribing of medications. Hence, we may have underestimated patterns of use for individuals who did not fill their prescriptions. Conversely, we may have overestimated patterns of use for individuals who were non-adherent to their medication regimen. We were also unable to capture individuals who may be using these drugs without a prescription. Finally, we imputed the date of conception based on claims for labor and delivery, which provide information on birth outcomes. Although this approach has been used previously, some degree of misclassification of pregnancy period is possible [19].

\section{Conclusion}

The present study evaluated real world dispensing patterns of benzodiazepines, both singularly and in combination with opioids, in a national sample of commercially insured pregnant women. Our findings indicate that as clinicians become increasingly cautious of prescribing opioids during pregnancy, benzodiazepine prescribing continues to increase. Our findings highlight the need for education and development of clinical guidelines for the safe use of benzodiazepines alone, and in combination with opioids, during pregnancy. Considering evidence suggesting the potentially teratogenic impact of these agents in combination, and especially given the paucity of safety evidence supporting their use, the persistent use of both agents, alone or in combination, is a health concern that warrants further attention.

\section{Abbreviations \\ NAS: Neonatal abstinence syndrome; FDA: Food and Drug Administration; CDC: Centers for Disease Control and Prevention; NBDP: National Birth Defects Prevention; OUD: Opioid Use Disorder}

\section{Supplementary Information}

The online version contains supplementary material available at https://doi. org/10.1186/s12884-021-03787-5.

Additional file 1. Temporal trends in pain diagnosis, cesarean delivery and anxiety diagnosis during pregnancy, 2007-2015. This figure displays 
temporal trends in pain diagnosis, cesarean delivery, and anxiety diagnosis among commercially insured pregnant women in the United States.

Additional file 2. Temporal trends for the five most commonly dispensed opioids during pregnancy, 2007-2015. This figure displays temporal trends in utilization of the five most commonly dispensed opioids during pregnancy among commercially insured pregnant women in the United States.

Additional file 3. Temporal trends for the five most commonly dispensed benzodiazepines during pregnancy, 2007-2015. This figure displays temporal trends in utilization of the five most commonly dispensed benzodiazepines during pregnancy among commercially insured pregnant women in the United States.

Additional file 4. Prevalence of the overall and the five most dispensed opioids before, during and after pregnancy, 2007-2015. This table displays prevalence in utilization of the five most commonly dispensed opioids before, during and after pregnancy among commercially insured pregnant women in the United States.

Additional file 5. Prevalence of the overall and the five most dispensed benzodiazepines before, during and after pregnancy, 2007-2015. This table displays prevalence in utilization of the five most commonly dispensed benzodiazepines before, during and after pregnancy among commercially insured pregnant women in the United States.

Additional file 6. Temporal trends in opioid dispensing and opioidbenzodiazepine co-dispensing in the post-delivery period by mode of delivery (cesarean delivery vs vaginal delivery) in the United States, 20072015. This figure displays the trends for opioid dispensing and opioidbenzodiazepine co-dispensing in the post-delivery period by mode of delivery among commercially insured women in the United States.

\section{Acknowledgements}

The statements, findings, conclusions, views, and opinions contained and expressed in this manuscript are based in part on data obtained under license from IQVIA ${ }^{\mathrm{TM}}$. Source: IQVIA ${ }^{\mathrm{TM}}$ Health Plan Claims Data January 2006 December 2015, IQVIA ${ }^{\mathrm{T}}$. All Rights Reserved. The statements, findings, conclusions, views, and opinions contained and expressed herein are not necessarily those of IOVIA ${ }^{\text {TM }}$ or any of its affiliated or subsidiary entities.

\section{Authors' contributions}

DMQ first conceived the study question, while both $\mathrm{DMQ}$ and $\mathrm{ABG}$ contributed to the development of the analysis plan and manuscript. ABG was primarily responsible for data analysis, under the mentorship of DMQ. All authors edited the manuscript text, ABG wrote the initial draft. DMQ supervised the work and both authors revised the manuscript critically for important intellectual content. DMQ was responsible for funding. Both DMQ and $A B G$ have reviewed the final manuscript and have approved the final version.

\section{Funding}

This study was sponsored by faculty support funding to Dr. Qato from the University of Maryland Baltimore and support as an ICTR KL2 Scholar from the University of Maryland Baltimore Institute for Clinical and Translational Research (1UL1TR003098-01). All authors report no relationship or financial interest with any entity that would pose a conflict of interest with the subject matter of this article. The sponsor had no role in the design of the study, the collection, analysis, and interpretation of data and in writing the manuscript. The submission of the study results was not contingent on the sponsor's approval or censorship of the manuscript.

\section{Availability of data and materials}

The data utilized in this analysis was obtained under license from IQVIA and is not publicly available.

\section{Declarations}

\section{Ethics approval and consent to participate}

This study received Institutional Review Board Approval from the University of Maryland, Baltimore. The statements, findings, conclusions, views, and opinions contained and expressed in this manuscript are based in part on data obtained under license from IQVIA ${ }^{\mathrm{TM}}$.

\section{Consent for publication}

Not applicable.

\section{Competing interests}

The authors report no competing interests.

Received: 23 June 2020 Accepted: 9 April 2021

Published online: 03 May 2021

\section{References}

1. Broussard CS, Rasmussen SA, Reefhuis J, Friedman JM, Jann MW, RiehleColarusso T, et al. Maternal treatment with opioid analgesics and risk for birth defects. Am J Obstet Gynecol. 2011;204(4):314. e1-e11.

2. Kellogg $\mathrm{A}$, Rose $\mathrm{CH}$, Harms $\mathrm{RH}$, Watson WJ. Current trends in narcotic use in pregnancy and neonatal outcomes. Am J Obstet Gynecol. 2011;204(3):259. e1-4.

3. Center for Substance Abuse T. SAMHSA/CSAT treatment improvement protocols. substance abuse treatment: addressing the specific needs of women. Rockville: Substance Abuse and Mental Health Services Administration (US); 2009.

4. Weismann D, Wilson J, Hagino O, Bennett D, Mulinare J, Depp R, et al. Use of psychoactive medication during pregnancy and possible effects on the fetus and newborn. Pediatrics. 2000;105(4 I):880-7.

5. ACOG Practice Bulletin. Clinical management guidelines for obstetriciangynecologists number 92, April 2008 (replaces practice bulletin number 87, November 2007). Use of psychiatric medications during pregnancy and lactation. Obstet Gynecol. 2008;111(4):1001-20.

6. Ogawa Y, Takeshima N, Furukawa TA. Maternal exposure to benzodiazepine and risk of preterm birth and low birth weight: a case-control study using a claims database in Japan. Asia Pac Psychiatry. 2018;10(3):e12309. https://doi. org/10.1111/appy.12309.

7. Tinker SC, Reefhuis J, Bitsko RH, Gilboa SM, Mitchell AA, Tran EL, et al. Use of benzodiazepine medications during pregnancy and potential risk for birth defects, National Birth Defects Prevention Study, 1997-2011. Birth Defects Res. 2019;111:613-20.

8. Vigod SN, Dennis C-L. Benzodiazepines and the Z-drugs in pregnancy - reasonably reassuring for neurodevelopment but should we really be using them? JAMA Network Open. 2019;2(4):e191430.

9. Wikner BN, Stiller CO, Bergman U, Asker C, Källén B. Use of benzodiazepines and benzodiazepine receptor agonists during pregnancy: neonatal outcome and congenital malformations. Pharmacoepidemiol Drug Saf. 2007;16(11): 1203-10. https://doi.org/10.1002/pds.1457.

10. Huybrechts KF, Bateman BT, Desai RJ, Hernandez-Diaz S, Rough K, Mogun H, et al. Risk of neonatal drug withdrawal after intrauterine co-exposure to opioids and psychotropic medications: cohort study. BMJ. 2017;358:j3326.

11. Seligman NS, Salva N, Hayes EJ, Dysart KC, Pequignot EC, Baxter JK. Predicting length of treatment for neonatal abstinence syndrome in methadone-exposed neonates. Am J Obstet Gynecol. 2008;199(4):396. e1-7.

12. Chan GM, Stajic M, Marker EK, Hoffman RS, Nelson LS. Testing positive for methadone and either a tricyclic antidepressant or a benzodiazepine is associated with an accidental overdose death: analysis of medical examiner data. Acad Emerg Med. 2006;13(5):543-7. https://doi.org/10.1197/j.aem.2 005.12.011.

13. Lee SC, Klein-Schwartz W, Doyon S, Welsh C. Comparison of toxicity associated with nonmedical use of benzodiazepines with buprenorphine or methadone. Drug Alcohol Depend. 2014;138:118-23. https://doi.org/10.101 6/j.drugalcdep.2014.02.014.

14. Bateman BT, Hernandez-Diaz S, Rathmell JP, Seeger JD, Doherty M, Fischer MA, et al. Patterns of opioid utilization in pregnancy in a large cohort of commercial insurance beneficiaries in the United States. Anesthesiology. 2014;120(5):1216-24. https://doi.org/10.1097/ALN.0000000000000172.

15. Desai RJ, Hernandez-Diaz S, Bateman BT, Huybrechts KF. Increase in prescription opioid use during pregnancy among Medicaid-enrolled women. Obstet Gynecol. 2014;123(5):997-1002. https://doi.org/10.1097/AOG. 0000000000000208.

16. Hanley GE, Mintzes B. Patterns of psychotropic medicine use in pregnancy in the United States from 2006 to 2011 among women with private 
insurance. BMC Pregnancy Childbirth. 2014;14(1):242. https://doi.org/10.11 86/1471-2393-14-242.

17. US Food and Drug Administration. FDA requires strong warnings for opioid analgesics, prescription opioid cough products, and benzodiazepine labeling related to serious risks and death from combined use 2016 Available from: https://www.fda.gov/news-events/press-announcements/ fda-requires-strong-warnings-opioid-analgesics-prescription-opioid-coughproducts-and-benzodiazepine.

18. Santo L, Rui P, Ashman JJ. Physician office visits at which benzodiazepines were prescribed: findings from 2014-2016 National Ambulatory Medical Care survey. Nat Health Stat Rep. 2020;137:1-16.

19. Ailes EC, Simeone RM, Dawson AL, Petersen EE, Gilboa SM. Using insurance claims data to identify and estimate critical periods in pregnancy: an application to antidepressants. Birth Defects Res A. 2016;106(11):927-34. https://doi.org/10.1002/bdra.23573.

20. CDC. Compilation of benzodiazepines, muscle relaxants, stimulants, zolpidem, and opioid analgesics with oral morphine milligram equivalent conversion factors. Atlanta: Centers for Disease Control and Prevention; 2017. Available from: https://www.cdc.gov/drugoverdose/resources/data. html

21. Sun EC, Dixit A, Humphreys K, Darnall BD, Baker LC, Mackey S. Association between concurrent use of prescription opioids and benzodiazepines and overdose: retrospective analysis. BMJ. 2017;356:j760.

22. Bateman BT, Mhyre JM, Hernandez-Diaz S, Huybrechts KF, Fischer MA, Creanga AA, et al. Development of a comorbidity index for use in obstetric patients. Obstet Gynecol. 2013;122(5):957-65.

23. Handal M, Engeland A, Rønning M, Skurtveit S, Furu K. Use of prescribed opioid analgesics and co-medication with benzodiazepines in women before, during, and after pregnancy: a population-based cohort study. Eur J Clin Pharmacol. 2011;67(9):953-60. https://doi.org/10.1007/s00228-011-1030-7.

24. Guy GP Jr, Zhang K, Bohm MK, Losby J, Lewis B, Young R, et al. Vital signs: changes in opioid prescribing in the United States, 2006-2015. MMWR Morb Mortal Wkly Rep. 2017;66(26):697-704. https://doi.org/10.15585/mmwr. mm6626a4.

25. Johnson K, Jones C, Compton W, Baldwin G, Fan J, Mermin J, et al. Federal response to the opioid crisis. Curr HIV/AIDS Rep. 2018;15(4):293-301. https:// doi.org/10.1007/s11904-018-0398-8.

26. Meisenberg BR, Grover J, Campbell C, Korpon D. Assessment of opioid prescribing practices before and after implementation of a health system intervention to reduce opioid overprescribing. JAMA Network Open. 2018; 1(5):e182908.

27. Parker AM, Strunk D, Fiellin DA. State responses to the opioid crisis. J Law Med Ethics. 2018;46(2):367-81. https://doi.org/10.1177/1073110518782946.

28. Osterman M, Martin J. Trends in low-risk cesarean delivery in the United States, 1990-2013. Nat Vital Stat Rep. 2014;63(6):1.

29. Paulozzi LJ, Mack KA, Hockenberry JM. Vital signs: variation among states in prescribing of opioid pain relievers and benzodiazepines-United States, 2012. MMWR Morb Mortal Wkly Rep. 2014;63(26):563-8.

30. Bais B, Molenaar NM, Bijma HH, Hoogendijk WJ, Mulder CL, Luik Al, et al. Prevalence of benzodiazepines and benzodiazepine-related drugs exposure before, during and after pregnancy: a systematic review and meta-analysis. J Affect Disord. 2020;269:18-27. https://doi.org/10.1016/j.jad.2020.03.014.

31. Dennis C-L, Falah-Hassani K, Shiri R. Prevalence of antenatal and postnatal anxiety: systematic review and meta-analysis. Br J Psychiatry. 2017;210(5): 315-23. https://doi.org/10.1192/bjp.bp.116.187179.

32. Hand DJ, Short VL, Abatemarco DJ. Substance use, treatment, and demographic characteristics of pregnant women entering treatment for opioid use disorder differ by United States census region. J Subst Abus Treat. 2017;76:58-63. https://doi.org/10.1016/j.jsat.2017.01.011.

33. Dowell D, Haegerich TM, Chou R. CDC guideline for prescribing opioids for chronic pain-United States, 2016. JAMA. 2016;315(15):1624-45. https://doi. org/10.1001/jama.2016.1464.

34. Campanelli CM. American Geriatrics Society updated beers criteria for potentially inappropriate medication use in older adults: the American Geriatrics Society 2012 beers criteria update expert panel. J Am Geriatr Soc. 2012;60(4):616.

35. Manchikanti L, Abdi S, Atluri S, Balog CC, Benyamin RM, Boswell MV, et al. American Society of Interventional Pain Physicians (ASIPP) guidelines for responsible opioid prescribing in chronic non-cancer pain: part 2--guidance. Pain Physician. 2012;15(3 Suppl):S67-116.
36. Ailes EC, Dawson AL, Lind JN, Gilboa SM, Frey MT, Broussard CS, et al. Opioid prescription claims among women of reproductive age-United States, 2008-2012. MMWR Morb Mortal Wkly Rep. 2015;64(2):37-41.

37. Lembke A, Papac J, Humphreys K. Our other prescription drug problem. N Engl J Med. 2018;378(8):693-5. https://doi.org/10.1056/NEJMp1715050.

38. Marc I, Toureche N, Ernst E, Hodnett ED, Blanchet C, Dodin S, et al. Mindbody interventions during pregnancy for preventing or treating women's anxiety. Cochrane Database Syst Rev. 2011;7:CD007559.

39. Shyken JM, Babbar S, Babbar S, Forinash A. Benzodiazepines in Pregnancy Clin Obstet Gynecol. 2019;62(1):156-67. https://doi.org/10.1097/GRF. 0000000000000417

40. Gopalan P, Glance JB, Azzam PN. Managing benzodiazepine withdrawal during pregnancy: case-based guidelines. Arch Womens Mental Health. 2014;17(2):167-70. https://doi.org/10.1007/s00737-013-0388-1.

\section{Publisher's Note}

Springer Nature remains neutral with regard to jurisdictional claims in published maps and institutional affiliations.
Ready to submit your research? Choose BMC and benefit from:

- fast, convenient online submission

- thorough peer review by experienced researchers in your field

- rapid publication on acceptance

- support for research data, including large and complex data types

- gold Open Access which fosters wider collaboration and increased citations

- maximum visibility for your research: over $100 \mathrm{M}$ website views per year

At BMC, research is always in progress.

Learn more biomedcentral.com/submissions 